masonem

\title{
Creación de la colección COVID-19 del Biobanco de Enfermedades Infecciosas en Argentina
}

\author{
Recibido: 2/12/20 Aceptado: 4/2/21
}

Yesica Longueira ${ }^{1,2}$, María Laura Polo ${ }^{1,2}$, Yanina Ghiglione ${ }^{1,2}$, Melina Salvatori ${ }^{1,2}$, Sabrina Azzolina ${ }^{1,2}$, Horacio Salomón ${ }^{1,3}$, María Florencia Quiroga ${ }^{1,3}$, Gabriela Turk $^{1,3}$ y Natalia Laufer ${ }^{1,3}$

\section{RESUMEN}

La pandemia de COVID-19 ha puesto en jaque a los sistemas de salud en el mundo; la vinculación entre la investigación biomédica y la práctica asistencial ha probado ser un requisito fundamental para dar respuesta a la misma de manera eficiente y rápida. En este sentido, los biobancos se constituyen como un componente clave ya que favorecen el almacenamiento de grandes volúmenes de muestras biológicas gestionadas en base a criterios que garanticen su óptima calidad, armonización y seguridad, respetando requisitos éticos y legales que aseguran los derechos de los ciudadanos. La cesión de estas muestras a distintos grupos de investigación promueve el desarrollo de nuevas herramientas diagnósticas y terapéuticas y vacunas. Frente a la llegada del SARS-CoV-2 a la Argentina, el Biobanco de Enfermedades Infecciosas estableció rápidamente la colección COVID-19 constituida por muestras de plasma, suero y células mononucleares de sangre periférica de personas cursando la enfermedad o recuperadas. En solo seis meses se enrolaron 825 donantes, lo que significa alrededor de 14.000 viales de material biológico almacenados y a disposición de los investigadores que lo soliciten. A tal efecto, se realizaron seis actos de cesión a diversos grupos pertenecientes a instituciones de investigación, mientras que tres se encuentran en evaluación. Las muestras cedidas han permitido, por ejemplo, el desarrollo de kits serológicos de producción nacional; lo que pone de manifiesto que el rápido establecimiento de esta colección, bajo un sistema de gestión eficiente, constituye una herramienta muy valiosa en la respuesta a esta nueva enfermedad.
${ }^{1}$ CONICET - Universidad de Buenos Aires. Instituto de Investigaciones Biomédicas en Retrovirus y SIDA (INBIRS), Paraguay 2155, piso 11, Ciudad Autónoma de Buenos Aires. Argentina.

${ }^{2}$ Facultad de Medicina, Universidad de Buenos Aires, Paraguay 2155, Ciudad Autónoma de Buenos Aires, Argentina.

${ }^{3}$ Departamento de Microbiología, Parasitología e Inmunología, Facultad de Medicina, Universidad de Buenos Aires, Paraguay 2155, Ciudad Autónoma de Buenos Aires, Argentina.

Autor para correspondencia: Mag. Yesica Longueira. Directora Científica Biobanco de Enfermedades Infecciosas (BBEI), Instituto de Investigaciones Biomédicas en Retrovirus y SIDA (UBA/CONICET). Paraguay 2155 Piso 11, CABA - C1121ABG - Argentina. Tel: +54 1145083689 int 113.

Email: ylongueira@fmed.uba.ar

Fuente de financiamiento: El presente trabajo recibe financiamiento por parte del Ministerio de Ciencia, Tecnología e Innovación (MINCyT) a través de un subsidio otorgado por la Agencia Nacional de Promoción de la Investigación, el Desarrollo Tecnológico y la Innovación (Agencia I+D+i) en la Convocatoria Extraordinaria COVID19 (Proyecto $\mathrm{N}^{\circ} 11$, IP N ${ }^{\circ}$ 285).

Conflicto de intereses: Los autores declaran que no tienen conflictos de intereses para reportar.

Los contenidos incluidos en este trabajo no han sido publicados anteriormente.

Palabras clave: Biobanco, COVID-19, bioespecímenes, investigación traslacional. 


\section{Introducción}

En diciembre de 2019, el nuevo coronavirus, SARS-CoV-2, fue el responsable de un aumento inusitado en los casos de neumonía en Wuhan, China (1), y, para el 11 de marzo de 2020 la Organización Mundial de la Salud (OMS) clasificó al COVID-19 (la enfermedad causada por el SARS-CoV-2) como una pandemia (2). Esta nueva enfermedad continúa expandiéndose por el planeta y hacia fines de octubre de 2020 ya lleva infectados más de 40 millones de personas y ha superado el millón de muertes en todo el mundo (3). Frente a este panorama, el manejo y prevención de esta infección se convirtió en el problema de salud pública más importante a nivel mundial, y el acceso a muestras biológicas en cantidad y calidad, una necesidad urgente. En este sentido, los biobancos son componentes clave para establecer puentes entre la investigación básica, traslacional y clínica, y la práctica asistencial, y al mismo tiempo son una herramienta para alcanzar la solución a esta pandemia.

Los biobancos se constituyen como la estructura óptima que favorece el almacenamiento de grandes volúmenes de muestras biológicas humanas gestionadas en base a criterios que garanticen su óptima calidad, armonización y seguridad, respetando en todo momento los requisitos éticos y legales que garantizan los derechos de los ciudadanos (4-7). En el contexto de una pandemia, como la causada por el virus SARS-CoV-2, el desarrollo de biobancos juega un papel central ya que permite: i) poner a disposición de los investigadores muestras biológicas humanas con la premura que la situación de emergencia sanitaria amerita, ii) garantizar la calidad de las muestras desde su obtención hasta el momento de la cesión, iii) almacenar y asociar información relevante de la propia muestra, iv) integrar datos sociodemográficos, epidemiológicos y clínicos asociados, facilitando de este modo la investigación de esta nueva enfermedad, v) garantizar que los requerimientos éticos y legales no sean vulnerados en un contexto de elevada presión sobre el sistema biomédico.

En Argentina, el primer caso de SARS-CoV-2 fue confirmado por el Ministerio de Salud el 3 de marzo de 2020 y se han reportado hacia fines de octubre más de 1 millón de casos, con más de 30.000 fallecidos (8). Ante este nuevo escenario, y de manera muy temprana, se tomaron una serie de medidas apuntadas a dar respuesta a esta situación. Por ejemplo, se estableció el aislamiento social preventivo y obligatorio (ASPO) que fue atravesando distintas fases, al mismo tiempo que se fue fortaleciendo el sistema de salud. Por su parte, desde el Ministerio de Ciencia, Tecnología e Innovación (MINCyT) se impulsó la creación de la Unidad Coronavirus (integrada también por el Consejo Nacional de Investigaciones Científicas y Técnicas -CONICET-y la Agencia Nacional de Promoción de la Investigación, el Desarrollo Tecnológico y la Innovación (Agencia I+D+i) (9). Esta unidad tiene por objetivo poner a disposición todas las capacidades de desarrollo de proyectos tecnológicos, recursos humanos, infraestructura y equipamiento que puedan ser requeridas para realizar tareas de diagnóstico e investigación sobre COVID-19. Una de las primeras medidas adoptadas por esta unidad fue el llamado a concurso de ideas-proyecto (IP) con la finalidad de seleccionar aquellas de mayor interés para el financiamiento de proyectos de investigación, desarrollo e innovación orientados a mejorar la capacidad nacional de respuesta a la pandemia en la Argentina.

El Biobanco de Enfermedades Infecciosas (BBEI) se creó en 2017 como un establecimiento dentro del Instituto de Investigaciones Biomédicas en Retrovirus y Sida (INBIRS), el cual depende de la Universidad de Buenos Aires y del CONICET. El objetivo del BBEl es poner a disposición de la comunidad científica muestras biológicas, asociadas a información clínica y epidemiológica de calidad, que sirvan de apoyo a la investigación, estableciéndose como el primer biobanco en Argentina de enfermedades infecciosas. Basándonos en los preceptos que i) el compromiso con el trabajo en colaboración y el intercambio de datos son esenciales para el avance del conocimiento sobre una base firme y de manera rápida, y que ii) el intercambio de muestras bien caracterizadas es un gran desafío a asumir para acelerar el desarrollo y la evaluación de distintos tipos de pruebas, es que desde el BBEI se propuso la creación de la colección de muestras COVID-19. La propuesta fue presentada a la convocatoria de financiamiento extraordinario impulsada por la Unidad Coronavirus del MINCYT bajo el título CREACION Y CARACTERIZACIÓN DE UNA COLECCIÓN DE MUESTRAS BIOLÓGICAS OBTENIDAS A PARTIR DE INDIVIDUOS POSITIVOS PARA LA INFECCIÓN POR SARS-COV-2 DENTRO DEL BIOBANCO DE ENFERMEDADES INFECCIOSAS (BBEI-COVID19) y fue uno de los 64 proyectos seleccionados entre más de 600 postulados.

Desde la llegada del nuevo coronavirus a la Argentina, los miembros del BBEI iniciamos la conformación de los documentos de la colección COVID-19, lanzamos la convocatoria a integrar la colección y realizamos la difusión 
del proyecto en medios públicos radiales, televisivos y redes sociales. Personas mayores de 16 años, de cualquier sexo, que presentaran diagnóstico de infección o sospecha de infección por SARS-CoV-2 fueron convocados a donar. Los casos confirmados o sospechosos debían cumplir con los criterios del Ministerio de Salud de la Nación (10). Aquellas personas que se encontraban en fase de convalecencia de la enfermedad realizaron su donación en las instalaciones del biobanco, mientras que para los casos agudos la toma de muestra se realizó en el centro de salud donde eran asistidos. Se han incluído también donaciones de contactos estrechos (convivientes) no infectados. En todos los casos los donantes consintieron en forma escrita la donación. La recogida sistemática de muestras comenzó el 9 de abril de 2020 y al 9 de octubre de 2020 cuenta con 825 donaciones.

\section{Materiales y métodos}

\section{Toma, procesamiento y almacenaje de muestras}

El BBEI se encuentra adscripto al Comité de Bioética de la Fundación Huésped. Para la recolección de las muestras se utilizó el consentimiento informado amplio del BBEI en su versión 3, aprobado por el Comité de Bioética. Un ejemplar en formato papel queda en poder del donante mientras que el biobanco conserva una copia en formato digitalizado (foto) según recomendación de la Sociedad Argentina de Infectología (11). La toma de muestra se realiza cumpliendo con los requisitos éticos establecidos por normativas nacionales e internacionales referidas a la investigación biomédica, la protección de datos de carácter personal y la bioética. En el caso de acuerdos entre el BBEl e instituciones de salud, se firma un acuerdo de almacenado y guarda entre las partes (involucrando comités de docencia y/o investigación según cada institución) a los fines de llevar adelante el enrolamiento de donantes. Actualmente tenemos acuerdos con cinco instituciones de salud que envían periódicamente muestras al BBEI.

El donante firma el consentimiento informado y si la muestra se toma en un centro de salud el médico o enfermero procede a la recolección de los datos clínicos en el formulario confeccionado a tal fin. En cambio, si la toma de la muestra se realiza de forma ambulatoria, algún miembro perteneciente al biobanco, de profesión médico, biólogo o bioquímico, es quien se encarga de la recolección de los datos. Luego se procede a la venopunción.
La toma de muestra consiste en $40 \mathrm{ml}$ de sangre entera: $30 \mathrm{ml}$ en tubos conteniendo EDTA y $10 \mathrm{ml}$ en tubos sin anticoagulantes. Las muestras se centrifugan a $2500 \mathrm{rpm}$ durante 10 minutos $y$, a partir de los primeros, se separan alícuotas de plasma mientras que de los segundos se separan alícuotas de suero. Luego, mediante la técnica de Ficoll-Hypaque ${ }^{\circledR}$ (GE Healthcare, UK) se obtienen las células mononucleares (linfocitos y monocitos) a partir de la fracción celular de la sangre. Una vez purificados, una fracción de dos millones de células mononucleares se guarda como pellet celulares (los cuales se pueden destinar, por ejemplo, a estudios genéticos o de transcriptómica), mientras que el resto se almacena en un medio de congelado que preserva la viabilidad celular. Este último tipo de muestra puede destinarse a estudios de, por ejemplo, funcionalidad inmune.

La recepción, procesamiento, almacenamiento y posterior cesión de muestras a investigadores solicitantes se realiza siguiendo los estándares establecidos en los manuales de procedimiento del BBEl, los cuales a su vez están basados en las normas de Buenas Prácticas Clínicas y de Laboratorio así como también las normas de procedimiento estandarizadas ISO 9001:2015.

Al ingreso, las muestras son identificadas por un código de donante denominado "Número de Biobanco", generado por el software NorayBanks ${ }^{\circledR}$ (V3.34, Noray Bioinformatics, Bizkaia, España). Una vez en el laboratorio, las muestras son alicuotadas y almacenadas en tubos con un "Tipo y Número de muestra" único. El investigador que solicita y adquiere las muestras solo tiene acceso a esta última identificación, lo que garantiza el cumplimiento de las normas de seguridad incluidas en la Ley 25.326 de Protección de Datos Personales, de la Dirección Nacional de Protección de Datos, y Ley 26.529 de Derechos del Paciente. Además, el INBIRS se encuentra inscripto en el Registro Nacional de Base de Datos (Registro No 100000271).

Las muestras se almacenan de manera de garantizar su trazabilidad y bajo condiciones que permitan su conservación a largo plazo. Así, suero, plasma y pellets celulares son almacenados en ultrafreezers a $-80{ }^{\circ} \mathrm{C}$, mientras que las células viables se almacenan en nitrógeno líquido a $-180^{\circ} \mathrm{C}$. Los datos clínicos, junto con datos inmunológicos, genéticos y virológicos que van generándose conforme se realiza la caracterización de la colección (ver apartado siguiente), también son almacenados apropiadamente. Bajo este sistema también 
se garantizan la confidencialidad de todos los flujos, de muestras y de información que se generen en torno al biobanco.

\section{Caracterización de las muestras de la colección}

Además de la creación de la colección COVID-19, el BBEI tiene por objetivo realizar una caracterización exhaustiva del perfil inmunológico general y específico, más una caracterización genética, sobre una subpoblación representativa de la colección.

1. Respuesta inmune celular: Como parte de la primera caracterización de la colección, se evaluaron los anticuerpos del tipo IgG e IgM contra SARS-CoV-2 en suero de cada donante mediante el ensayo de ELISA utilizando el kit COVIDAR (ELISA cualitativo). En un subgrupo de muestras, se evaluó, además, el título de anticuerpos específicos (ELISA cuantitativo).

2. Análisis del fenotipo inmune celular total y específico para SARS-CoV-2: Se realizó una caracterización exhaustiva de las diferentes poblaciones (LTh1, LTh17, Tfh, Ltregs, monocitos, células productoras de anticuerpos, células NK, entre otras) de leucocitos en sangre periférica mediante citometría de flujo. En una segunda etapa se evaluará la funcionalidad de la respuesta de linfocitos T CD4 y CD8 específicos contra el virus a través de las técnicas citometría de flujo y ELISPOT.

3. Se evaluaron los niveles plamáticos de diferentes mediadores solubles a través de inmunoensayos multiparamétricos por citometría de flujo con el objetivo de determinar los niveles de inflamación en cada una de las muestras.

4. Análisis genómico mediante el uso de un microarray específicamente diseñado para estudios relacionados a SARS-CoV-2 (Axiom Human Genotyping SARS-CoV-2 Research Array). El mismo incluye la tipificación molecular de los antígenos leucocitarios humanos (HLA), polimorfismos del receptor ACE2, de receptores $\mathrm{KIR}$, entre otros.

\section{Resultados}

\section{Descripción de la colección COVID-19}

La colección COVID-19 del BBEl se puso en marcha el 27 de marzo de 2020 con tareas administrativas y de gestión realacionadas con la nueva colección. El 9 de abril de 2020

\section{Figura 1. Número de donantes incluídos en la colección COVID-19 en función del tiempo}

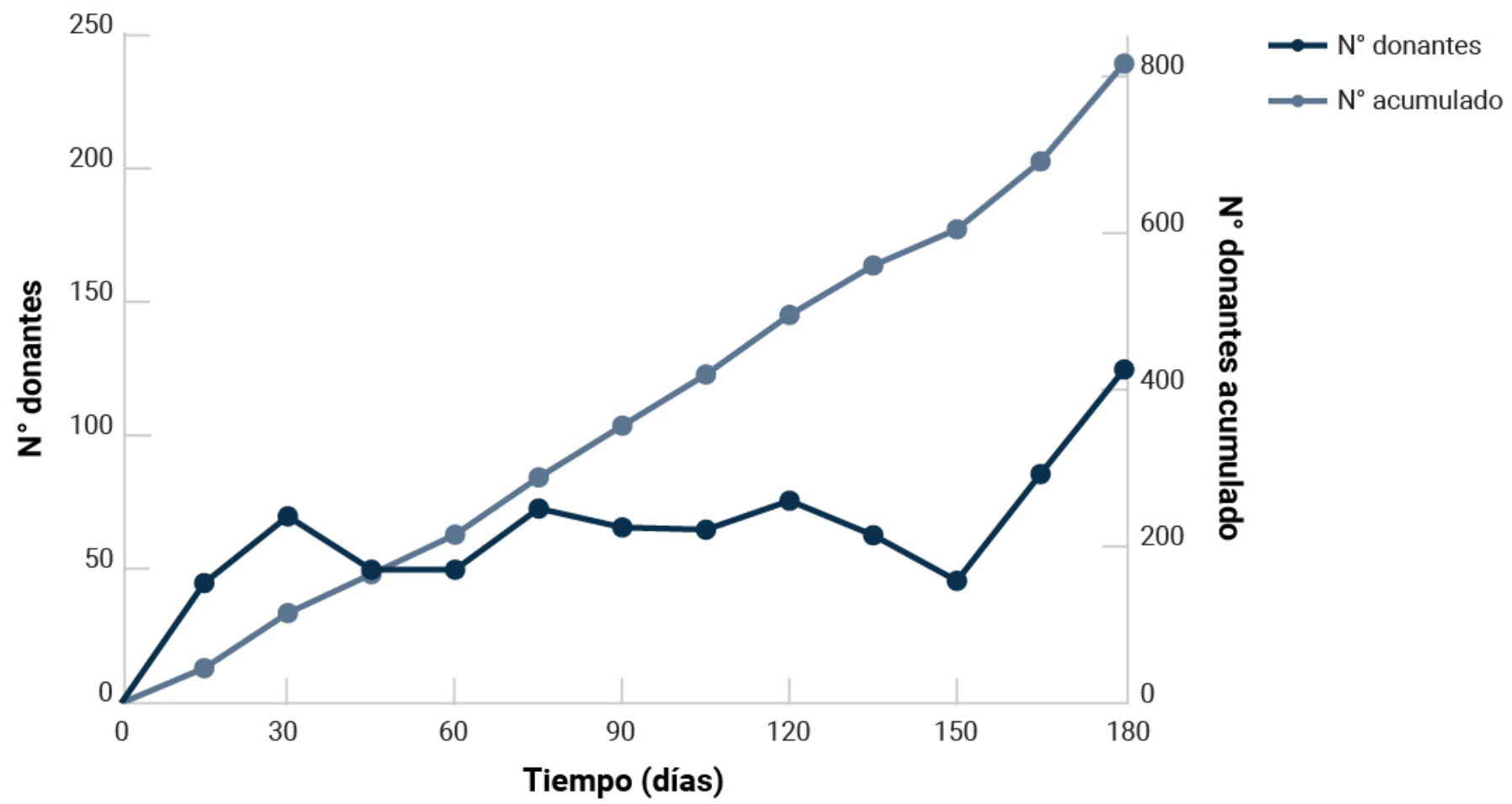


se recibieron las primeras tres muestras. Al 9 de octubre de 2020 se han recibido 825 donaciones, un promedio de 6,65 muestras/día hábil, lo que representa el $82,5 \%$ de reclutamiento total estipulado en el proyecto $(\mathrm{N}=1000)$ en solo seis meses (Figura 1). Además, en 34 casos, los donantes donaron más de una vez.

El $57,1 \%(n=471)$ son mujeres, mediana de edad 41 años (RIQ 32-53 años), 6,6\% corresponden a donantes de menos de 25 años, y $16,8 \%$ a donantes de más de 60 años. El 17,6\% de las donaciones corresponden a individuos cursando la fase aguda de la infección, $71,6 \%$ a convalecientes, 5,5\% a convivientes no infectados, $1,9 \%$ a individuos con dos episodios confirmados de COVID-19 y 2,8\% se encuentran pendientes de clasificación. En el 19,9\% los donantes presentaron un cuadro grave de COVID-19 y en el $80,1 \%$ leve a moderado. En el $84,1 \%$ de los casos el contagio se produjo en forma local. Cabe destacar que, durante los primeros meses de enrolamiento, la mayoría de los casos estaban representados por donantes que habían adquirido la infección en el exterior del país; esta proporción se fue invirtiendo con el correr de las semanas conforme la epidemia fue avanzando en el territprio y se fueron dando restricciones de las fronteras a nivel mundial.

De las 825 donaciones se obtuvieron, tras el procesamiento

\section{Figura 2. Solicitudes y cesiones de muestras a investigadores solicitantes}

\section{Solicitudes en proceso}
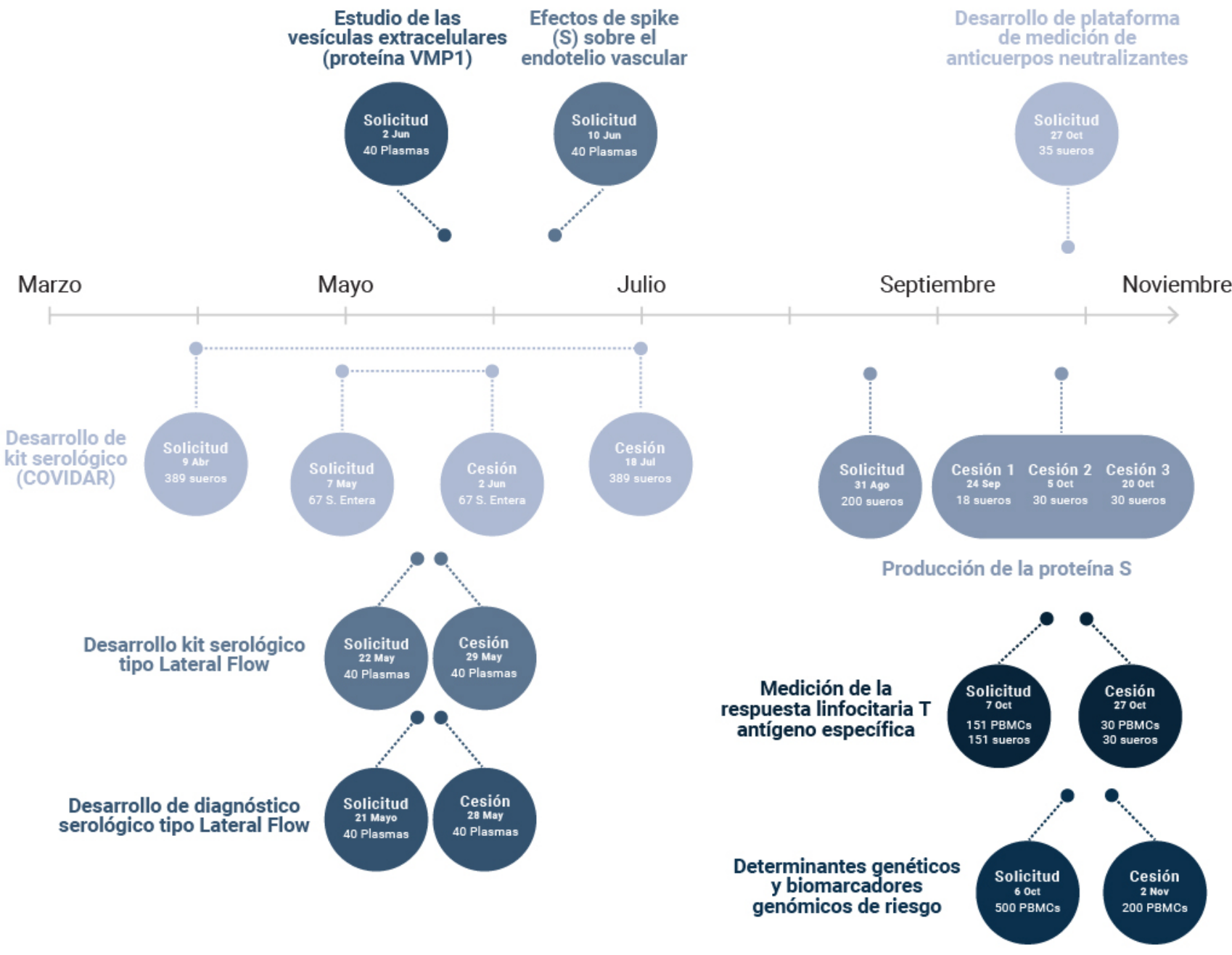

Solicitudes ejecutadas 
de las muestras de sangre entera, 5596 viales de $1 \mathrm{~mL}$ de plasma y 2287 de suero, 1616 pellets celulares y 4347 viales de, en promedio, 9 millones de células mononucleares de sangre periférica cada uno. Sobre este último material se realizaron pruebas de control de calidad, pudiéndose corroborar en promedio una recuperación del $80 \%$ de las células iniciales, con un $95 \%$ de viabilidad.

De los especímenes almacenados, 892 viales se han utilizado para la primera caracterización de la colección, analizando anticuerpos específicos del tipo IgG e IgM y titulando los anticuerpos del tipo IgG mediante el ensayo de ELISA. Además, se titularon anticuerpos con capacidad neutralizante frente al virus SARS-CoV-2 salvaje (cepa INVIV8 gentilmente cedida por la Dra. Sandra Gallego, Instituto de Virología Dr. José María Vanella, UNC). Sobre un $n=236$ muestras, se evaluaron los niveles plasmáticos de 13 moléculas relacionadas con los niveles de activación inmune e inflamación: CXCL10, CCL2, CXCL9, CXCL8, CD14s, Rantes, IL-17A, IFN-g, TNF-a, IL-10, IL-6, IL-4 e IL-2. La caracterización del inmunofenotipo inmune, así como el análisis genético, se encuentran en proceso.

Uno de los principales objetivos del biobanco es nutrir a desarrollos o proyectos de investigación con las muestras de la colección y sus datos asociados. Aquellos investigadores que requieren muestras lo solicitan a través de un correo electrónico al biobanco y reciben como respuesta un formulario de solicitud de muestras. Dicho formulario debe ser reenviado por el investigador de manera completa junto a la aprobación del proyecto por parte del comité de ética de su centro de investigación. A continuación, el director del biobanco se encarga de hacerlo llegar a los miembros del comité de ética y del comité científico, quienes harán la evaluación del mismo y decidirán si se aprueba o no la cesión. En este sentido, se cedieron hasta la fecha 844 viales para seis proyectos de investigación de universidades públicas, mientras que otros 278 se cederán en futuros actos a los mismos grupos en función de los resultados preliminares que vayan arrojando cada una de las investigaciones. Actualmente, tres proyectos adicionales se encuentran en proceso de evaluación. En todos los casos, el tiempo transcurrido entre la solicitud de muestras y la cesión fue menor a 30 días (Figura 2).

\section{Discusión}

Durante las últimas décadas, los eventos de emergencia y reemergencia de patógenos virales han aumentado en frecuencia y gravedad. La constante vigilancia epidemiológica es fundamental para dar una respuesta rápida a cada uno de estos eventos. En este sentido, el desarrollo rápido, la evaluación y distribución de ensayos diagnósticos que logren identificar patógenos con precisión y rapidez son componentes críticos de esta respuesta. Por otro lado, el desarrollo de terapias específicas y vacunas preventivas completan el cuadro. La pandemia de COVID-19 es un hecho sin precedentes en el mundo moderno y está poniendo a prueba a los distintos sistemas de salud, sistemas científicos y a la sociedad entera. En este contexto, existen numerosos ejemplos en los que el trabajo mancomunado y colaborativo junto con el intercambio de datos y recursos, está dando sus frutos en la lucha contra la pandemia. Sin embargo, aún quedan diversos desafíos por atravesar (12). Uno de ellos está constituido por la necesidad de establecer biobancos sostenibles para la recopilación, caracterización y archivo estandarizados de muestras, y compartir estas muestras para facilitar y acelerar el desarrollo de estrategias de diagnóstico, tratamiento y prevención. Este desafío es particularmente crítico en Argentina, donde el concepto de biobanco no está instalado ni siquiera en la comunidad biomédica (13).

En este sentido, desde el BBEl entendimos que la actual situación epidemiológica/sanitaria constituye una oportunidad que no debía desaprovecharse, y asumimos el desafío de manera temprana mediante la creación de la colección BBEI-COVID19, entendiéndola como una herramienta fundamental para estudios de biomarcadores, factores de riesgo, identificación de blancos terapéuticos y vacunas, entre otros, así como también para entender epidemias pasadas (SARS-CoV-1 y MERS-CoV) y futuras por otros coronavirus. Rápidamente, la colección COVID-19 ha puesto a disposición de la ciencia numerosos bioespecímenes junto a sus respectivos datos demográficos y clínicos, donde están representados los distintos tipos de evolución de la enfermedad (asintomáticos, leve, moderado, severo) en proporciones acordes a datos poblacionales. Esto permitió poder arribar a resultados confiables en el menor tiempo posible, lo cual es extremadamente relevante en un contexto de pandemia. Un ejemplo fue el desarrollo de un test serológico nacional en tan solo 45 días desde la declaración del ASPO que dispuso el Gobierno Nacional gracias, en gran parte, a la cesión de muestras y datos provistos por el BBEI. Dicho ensayo permite determinar la presencia de anticuerpos del tipo IgG e IgM contra SARSCoV-2 en sueros de pacientes que cursaron la enfermedad 
COVID-19. Poco tiempo después de su aprobación ante la Administración Nacional de Medicamentos, Alimentos y Tecnología Médica, el grupo de investigación recurrió al biobanco solicitando muestras pareadas de suero y sangre entera para la validación del ensayo desarrollado con muestras de sangre obtenidas por punción digital $(14,15)$.

La rápida instauración de la colección fue posible gracias a un número de factores que facilitaron el proceso. Entre ellos, cabe mencionar que el INBIRS cuenta con la infraestructura, equipamiento y recursos (especialmente aquellos relacionados con la bioseguridad) que permiten manipular y almacenar las muestras mencionadas. También, para garantizar la gestión eficiente y segura de las muestras biológicas y sus datos asociados, el BBEl contaba con el software NorayBanks ${ }^{\circledR}$ (V3.34, Noray Bioinformatics, Bizkaia, España), sistema fundamental para asegurar la calidad, trazabilidad y confidencialidad de la preservación, análisis, almacenamiento y distribución del material biológico y de su información asociada, lo cual resultó un factor imprescindible. Además, la obtención de financiamiento e, indirectamente, el apoyo institucional a la iniciativa fueron clave para poder afianzar una propuesta completa. A su vez, el proyecto se vio enriquecido con la incorporación de recursos humanos provenientes de distintas áreas, como son la investigación básica y la construcción de nuevas redes de colaboración con distintos efectores de salud.

Sin embargo, durante el proceso también hubo diversos desafíos. Tal vez el más importante fue cómo tratar de interaccionar con un sistema de salud en crisis y en proceso de reconstrucción, para lograr reclutamiento de los donantes. Una salida a esto fue la apertura de la convocatoria directamente a la comunidad, independizándonos parcialmente de las instituciones de salud. Para esto, se hizo una fuerte campaña de difusión en los medios de prensa y en redes sociales apelando a la sensibilidad de las personas que habían atravesado la enfermedad y convocándolas a donar. Esto implicó un esfuerzo extra porque la formación de la mayor parte de los integrantes del proyecto no se relaciona con las ciencias sociales e implicó poder aprender a transmitir los mensajes en forma clara y sencilla. Además implicó una reestructuración de los canales normales de trabajo, ya que investigadores básicos debieron ser los responsables del contacto con los donantes y de la obtención de las muestras. Sin embargo, trajo como resultado el estrechamiento de la brecha entre al menos un componente del sistema científico y la comunidad, y viceversa.
En resumen, la rápida creación de la colección COVID-19 del BBEI representa una herramienta clave para la pandemia a nivel local, afianzando los lazos entre la investigación básica y traslacional y permitiendo aportar un beneficio a la comunidad en el corto y mediano plazo.

\section{Reconocimientos}

Los miembros del BBEl agradecemos a todos los donantes que participan de la colección por su invaluable colaboración con esta iniciativa. Asimismo, agradecemos a los miembros del comité científico del BBEl por su colaboración en la evaluación de los proyectos solicitantes, a los investigadores que solicitaron muestras al BBEl, a las autoridades del INBIRS por su apoyo al proyecto, a Fernando Montesano por su colaboración en tareas administrativas y a todos aquellos que colaboraron con la difusión de la convocatoria. 


\section{Bibliografía}

1. Zhu H, Wei L, Niu P. 2020. The novel coronavirus outbreak in Wuhan, China. Glob Health Res Policy 5:6.

2. World Health Organization Coronavirus disease (COVID-19) situation reports. 2020. https:// wwwwhoint/emergencies/diseases/novelcoronavirus-2019/situation-reports.

3. Coronavirus Resource Center. 2020. https:// coronavirusjhuedu/maphtml.

4. Doménech García N, Cal Purrinos N. 2014. Biobancos y su importancia en el ámbito clínico y científico en relación con la investigación biomédica en Espa na. Reumatol Clin 10:304-308.

5. Malsagova K, Kopylov A, Stepanov A, Butkova T, Sinitsyna A, Izotov A, et al. 2020. Biobanks-A Platform for Scientific and Biomedical Research. Diagnostics (Basel) 10.

6. Artene SA, Ciurea ME, Purcaru SO, Tache DE, Tataranu LG, Lupu M, et al. 2013. Biobanking in a constantly developing medical world. ScientificWorldJournal 2013:343275.

7. Kinkorova J, Topolcan 0. 2018. Biobanks in Horizon 2020: sustainability and attractive perspectives. EPMA J 9:345-353.

8. Ministerio de Salud de la Republica Argentina. Daily Report. 2020. https://wwwargentinagobar/ coronavirus/informes-diarios/reportes/julio2020.

9. Ministerio de Ciencia, Tecnología e Innovacion de la Republica Argentina. 2020. https:// wwwargentinagobar/ciencia/unidad-coronavirus.

10. Ministerio de Salud de la Republica Argentina. Definición de caso COVID19. 2020. https:// wwwargentinagobar/salud/coronavirus-COVID-19/ definicion-de-caso.

11. Sociedad Argentina de Infectologia. 2020. https://wwwsadiorgar/guias-recomendacionesy-consensos/item/1292-recomendaciones-ciensayos-clinicos-covid-19.

12. Peeling RW, Boeras D, Wilder-Smith A, Sall A, Nkengasong J. 2020. Need for sustainable biobanking networks for COVID-19 and other diseases of epidemic potential. Lancet Infect Dis 20:e268-e273.

13. Longueira Y. 2018. Evaluación del conocimiento en la comunidad médica de las características y alcance de los biobancosUniversidad Católica de Valencia San Vicente Mártir, España.

14. Consejo Nacional de Investigaciones Científicas y Técnicas CONICET. 2020. https://wwwconicetgovar/ tag/covidar-igg/.
15. Ojeda DS, Lopez Ledesma MMG, Pallares H, Costa Navarro GS, Sanchez L, Perazzi B, et al. 2020. Emergency Response for Evaluating SARS-CoV-2 Immune Status, Seroprevalence and Convalescent Plasma in Argentina. medRxiv doi:10.1101/2020.10. 21.20216960:2020.10.21.20216960. 
Establishment of a COVID-19 collection within the Biobank of Infectious Diseases in Argentina

The COVID-19 pandemic has driven an unprecedented health crisis. Cooperation between biomedical research and healthcare practice has been shown to be a fundamental requirement to provide an efficient and timely response. In this regard, biobanks are key components since they allow the storage of large volumes of biological samples with guaranteed optimum quality, harmonization and safety, ensuring ethical and legal requirements which protect citizen rights. The transfer of these samples to different research groups fosters the development of new diagnostic and therapeutic tools as well as vaccines. Upon SARS-CoV-2 arrival to Argentina, the Biobank of Infectious Diseases rapidly established the COVID-19 collection comprised by plasma, serum and peripheral blood mononuclear cells samples obtained from people within the acute phase of the infection or who have already recovered. In only 6 months, 825 donors were enrolled, representing around 14,000 vials of biological material stored and available to researchers who might require it. In this line, 6 transfer agreements have been already performed to different groups belonging to national research institutions, while 3 are under evaluation. The transferred samples have allowed, for instance, the development of nationally produced serologic kits, which shows that the rapid establishment of this collection, under an efficient management system, represents a highly valuable tool in the response to this new disease.

Keywords: Biobank, COVID-19, biospecimens, translational research. 